\title{
Sensitized Emission from a Chemotherapeutic Drug Conjugated to CdSe/ZnS QDs
}

\author{
S. Shankara Narayanan, Sudarson Sekhar Sinha, and Samir Kumar Pal* \\ Unit for Nano Science \& Technology, Department of Chemical, Biological \& Macromolecular Sciences, \\ S. N. Bose National Centre for Basic Sciences, Block JD, Sector III, Salt Lake, Kolkata 700 098, India
}

Received: April 15, 2008; Revised Manuscript Received: May 28, 2008

\begin{abstract}
Picosecond-resolved photoluminescence (PL) and polarization gated PL anisotropy measurements have been performed to confirm a strong sensitized emission from a chemotherapeutic drug, Merocyanine 540 (MC540), conjugated to highly luminescent $\mathrm{CdSe} / \mathrm{ZnS}$ quantum dots (QDs) via Förster resonance energy transfer (FRET) from QDs (donor) to the drug molecule (acceptor). Both steady-state and time-resolved PL measurements clearly confirm FRET to be the reason behind the PL quenching of the QDs (energy donor) in the QD-drug complex. The picosecond-resolved PL transients of QD-drug complex monitored at the donor $(500 \mathrm{~nm})$ and the acceptor peak emission $(620 \mathrm{~nm})$ wavelengths revealed a fast decay time for the donor $(500 \mathrm{~nm})$ and a rise component of similar time scale for the acceptor $(620 \mathrm{~nm})$. The observation of similar decay and rise times for the donor and the acceptor, respectively, in the QD-MC540 system gave a clear signature of the sensitized emission from MC540. Further confirmation of sensitization has been obtained from the picosecondresolved anisotropy measurements, which revealed a fast component indicating efficient energy transfer from dipolar QD excitons to the conjugated drug molecules. It is anticipated that the present characterization of sensitized emission from a donor-acceptor pair formed by chemotherapeutic drugs and QDs can be applied to other systems as well for the confirmation of sensitized emission.
\end{abstract}

\section{Introduction}

Over the past decade, quantum dots (QDs) have been increasingly used in a wide range of applications in biomedicine. ${ }^{1-3}$ In this context, several groups have recently addressed the potential of using QDs as photosensitizers and phototherapeutic agents. ${ }^{4-7}$ For example, Burda and co-workers ${ }^{4}$ have demonstrated the applicability of CdSe QDs for photodynamic therapy through the activation of a photosensitizer (Pc4) by QDs via Förster resonance energy transfer (FRET). Shi et al. ${ }^{6}$ prepared biocompatible QD-organic dye nanocomposites, which could generate singlet oxygen enough for biological applications. In another similar study, CdSe QD-phthalocyanine (Pc) conjugates were shown to be an excellent system to perform near-infrared excitation of Pc molecules via two-photon sensitization of the QD followed by energy transfer (ET) to the Pc. ${ }^{8}$ The ET efficiency as a function of QD size under different surface chemistry conditions was also further investigated. ${ }^{9}$

So far in the FRET studies of a QD-based energy donor-acceptor (DA) system in which QD acts as a donor, the sensitized emission from the acceptor (dye or dye-labeled protein/DNA) has been established from the steady-state emission or timeresolved fluorescence/transient absorption studies only. ${ }^{8-10}$ For example, Weiss et al. ${ }^{11}$ have synthesized water-stable peptidecoated CdSe QD-photosensitizer conjugates and used them to efficiently generate singlet oxygen via indirect excitation through FRET from the QDs to photosensitizers or by direct excitation of the photosensitizers. They confirmed FRET using both steadystate and time-resolved studies. Iridium complex-modified $\mathrm{CdSe} /$ $\mathrm{ZnS}$ QDs ${ }^{12}$ have been designed in which QDs acts as an imaging center, while the Ir complex acts as a sensitizer whose sensitized emission in the conjugate was established through the steadystate emission alone. Whereas in a recent study ${ }^{13}$ on energy

* To whom correspondence should be addressed. E-mail: skpal@ bose.res.in. Fax: 913323353477. transfer between $\mathrm{CdSe} / \mathrm{ZnS}$ QDs and fluorescent proteins, the sensitized emission from the protein was detected by monitoring both the steady-state emission and anisotropy of the nanobioconjugate system. The shortening of the fluorescence lifetime of the donor-acceptor system with respect to the donor alone was also evident in both the studies described above. However, the shortening of the fluorescence lifetime in a DA system may not always ensure sensitization of the attached acceptor moiety due to possibility of other nonradiative processes in the QDs ${ }^{14}$ (in addition to energy transfer).

In the present work, we report that, in a FRET-based DA system, the sensitized emission from the acceptor could be characterized by a fast component of the donor photoluminescence (PL) decay together with a corresponding rise component of similar time scale for the acceptor. To the best of our knowledge, there are no reports that have shown the sensitized emission from a drug conjugated to QDs through a rise component at the emission peak of acceptor via time-resolved PL studies, which is the motivation of our present work. In particular, for our studies we have used a highly luminescent surface-passivated (TOPO-capped) core-shell CdSe/ZnS QDs in toluene as the energy donor, which has a PL peak at 520 $\mathrm{nm}$, and an anionic cyanine chemotherapeutic drug, merocyanine-540 (MC540) as the energy acceptor. MC540, an anionic cyanine dye, is a heterocyclic chromophore (Scheme 1) that is commonly used as a sensitizer in chemotherapy and, more recently, as a sensitizer in photoelectrochemical cells for solar energy conversion. ${ }^{15}$ The strong sensitized emission of the drug in the QD-drug complex has been confirmed from the PL lifetime measurements in the form of a rise component at the acceptor side. We have also undertaken time-resolved anisotropy studies of the QD-drug complex, which reveal a fast component indicating efficient energy transfer from dipolar QD excitons to the conjugated drug molecules leading to sensitized emission 


\section{SCHEME 1: Chemical Structure of Merocyanine 540}

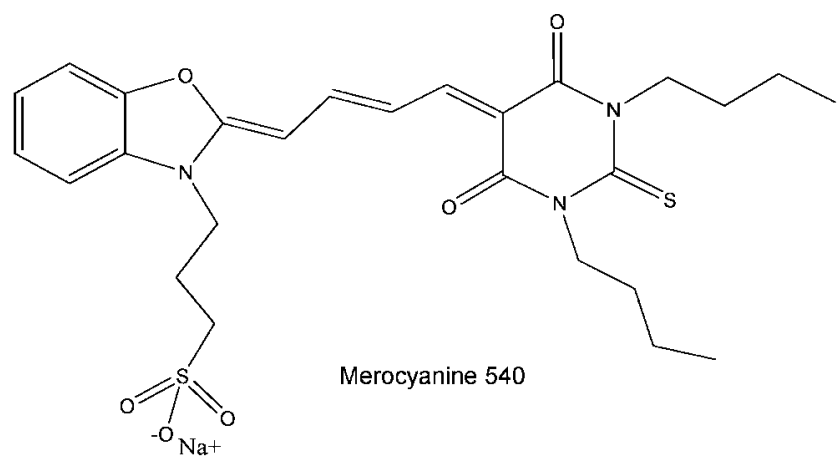

SCHEME 2: Binding of Drug Molecules (MC540) to the Surface of CdSe/ZnS QDs due to Strong Affinity of Sulfur Atom of MC540 Compared to the Oxygen Atom of the TOPO on the Surface of QDs

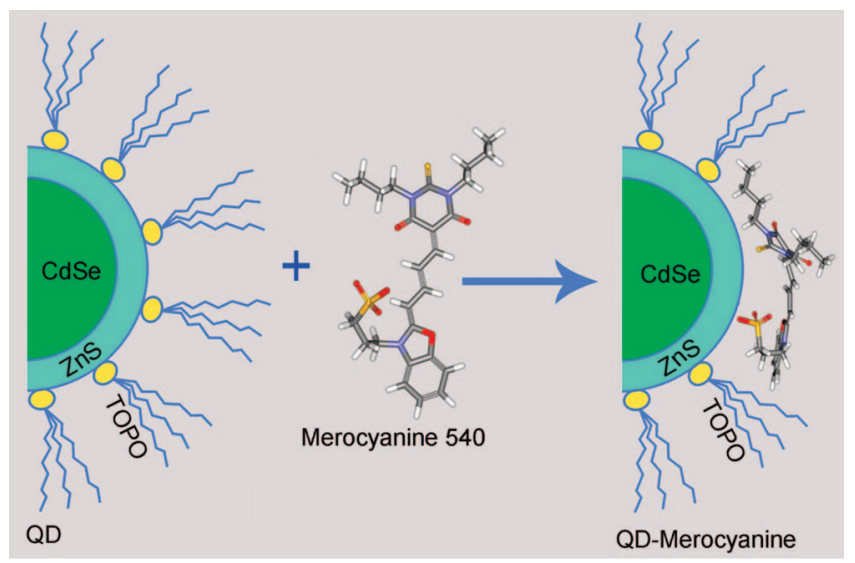

from the drug. Confirmation of energy migration through timeresolved fluorescence anisotropy is also scarce in the literature. ${ }^{16,17}$

\section{Materials and Methods}

TOPO-capped core-shell CdSe/ZnS QDs in toluene (Adirondack Green) having a core diameter of $2.1 \mathrm{~nm}$ (shell thickness $\sim 0.3 \mathrm{~nm}$ ) and a PL peak at $520 \mathrm{~nm}$ were obtained from Evident Technologies, Inc. (New York). Merocyanine 540, 2-[4-(1,3-dibutyltetrahydro-4,6-dioxo-2-thioxo-5(2H)-pyrimidinylidene)-2-butenylidene] sodium (MC540) and toluene were obtained from Sigma. QD-MC540 complex was prepared by simple overnight stirring of the QDs with MC540 in toluene followed by sonication and filtration to remove any undissolved MC540 in the solution. Because of the high affinity of $\mathrm{ZnS}$ to the sulfur compared to oxygen, it is expected that, on mixing CdSe/ZnS QDs with MC540, the TOPO ligands around the QDs will be replaced by MC540 due to the presence of sulfur atoms in it. We expect that the direct binding of MC540 to the QD surface can take place through the thionyl sulfur atom attached to heterocyclic group providing solubility in toluene. In addition, the sulfur atom of the sulfonate group $\left(\mathrm{SO}_{3}{ }^{-}\right)$with its counterion $\mathrm{Na}^{+}$could also serve as a binding site to the nanocrystal surface (Scheme 2). A direct binding of MC540 molecules to the surface of $\mathrm{TiO}_{2}$ nanoparticles through the direct attachment of sulfur atom of MC540 has been proposed by Kamat and co-workers. ${ }^{18}$ It was found that the conjugation of QDs to MC540 did not affect the spectroscopic properties of merocyanine as indicated by negligible shift in the absorption and emission spectra of QD-MC540 complex compared to MC540 alone (data not shown). The above observation may indicate nonspecific at- tachment of MC540 to the surface of QDs instead of covalent conjugation. The final QD-drug complex was found to be stable in the solution. The stock solution of the complex was diluted in toluene to a level such that the effects of absorption of the incident light, reabsorption of PL, and aggregation of nanoparticles were negligible.

Steady-state absorption and emission spectra were measured using the Shimadzu model UV-2450 spectrophotometer and Jobin Yvon Model Fluoromax-3 fluorometer, respectively. A FEI Tecnai high-resolution transmission electron microscope (HRTEM) equipped with an energy-dispersive X-ray (EDAX) spectrometer was used to characterize the internal structures of samples and to analyze their elemental composition. For the TEM measurements, QD sample was dispersed in toluene, sonicated for $8 \mathrm{~min}$, and then drop coated over a carbon-coated grid ( $\mathrm{Cu}$ mesh 200). Fluorescence transients were measured and have been fitted by using a commercially available spectrophotometer (LifeSpec-ps) from Edinburgh Instrument (excitation wavelength $375 \mathrm{~nm}, 80$-ps instrument response function (IRF)). The observed fluorescence transients are fitted by using a nonlinear least-squares fitting procedure to a function $(X(t)=$ $\left.\int_{0}^{\mathrm{t}} E\left(t^{\prime}\right) R\left(t-t^{\prime}\right) \mathrm{d} t^{\prime}\right)$ comprising a convolution of the $\operatorname{IRF}(E(t))$ with a sum of exponentials $\left(R(t)=A+\sum_{\mathrm{i}=1}^{\mathrm{N}} B_{\mathrm{i}} \mathrm{e}^{-t / \tau_{\mathrm{i}}}\right)$ with preexponential factors $\left(B_{\mathrm{i}}\right)$, characteristic lifetimes $\left(\tau_{\mathrm{i}}\right)$, and a background $(A)$. Relative concentration in a multiexponential decay is finally expressed asfollows: $c_{n}=B_{n} / \sum_{\mathrm{i}=1}^{\mathrm{N}} B_{\mathrm{i}} \times 100$. The quality of the curve fitting is evaluated by reduced $\chi^{2}$ and residual data. For temporal fluorescence anisotropy measurements, emission polarization was adjusted to be parallel or perpendicular to that of the excitation and anisotropy was defined as $r(t)=\left[I_{\text {para }}-G I_{\text {perp }}\right] /\left[I_{\text {para }}+2 G I_{\text {perp }}\right]$. The magnitude of $G$, the grating factor of the emission monochromator of the TCSPC system, was determined following longtime tail matching technique. ${ }^{19}$

In order to estimate the FRET efficiency of the donor (QD) to the acceptor (MC540) and hence to determine distances of donor-acceptor pairs, we have followed the methodology described in chapter 13 of ref 20 . The Förster distance $\left(R_{0}\right)$ is given by

$$
R_{0}=0.211\left[\kappa^{2} n^{-4} Q_{\mathrm{D}} J(\lambda)\right]^{1 / 6} \text { (in } \AA \text { ) }
$$

where $\kappa^{2}$ is the orientation factor. In the present study $\kappa^{2}=$ $2 / 3$, a value for randomly oriented dipoles, is assumed for FRET in our QD-dye conjugates as described by Weiss et al. ${ }^{11}$ The refractive index $(n)$ of the medium (toluene) is $1.496 . Q_{\mathrm{D}}$ is the quantum yield of the donor in the absence of acceptor $(0.5)$ and $J(\lambda)$, the overlap integral, which expresses the degree of spectral overlap between the donor emission and the acceptor absorption. Knowing the value of $R_{\mathrm{o}}$, the donor-acceptor distance $(r)$ can easily be calculated using the formula,

$$
r^{6}=\left[R_{\mathrm{o}}{ }^{6}(1-E)\right] / E
$$

Here $E$ is the efficiency of energy transfer. The transfer efficiency $(E)$ is measured using the relative lifetime of the donor in the absence $\left(\tau_{\mathrm{D}}\right)$ and presence $\left(\tau_{\mathrm{DA}}\right)$ of the acceptor.

$$
E=1-\left(\tau_{\mathrm{DA}} / \tau_{\mathrm{D}}\right)
$$

It has to be noted that eq 3 holds rigorously only for a homogeneous system (i.e., identical donor-acceptor complexes) in which the donor and the donor-acceptor complex have single-exponential decays. However, for donor-acceptor systems decaying with multiexponential lifetimes, ${ }^{20}$ energy-transfer efficiency $(E)$ is calculated from the amplitude weighted lifetimes $\langle\tau\rangle=\sum_{i} \alpha_{i} \tau_{i}$ where $\alpha_{i}$ is the relative amplitude 
(a)

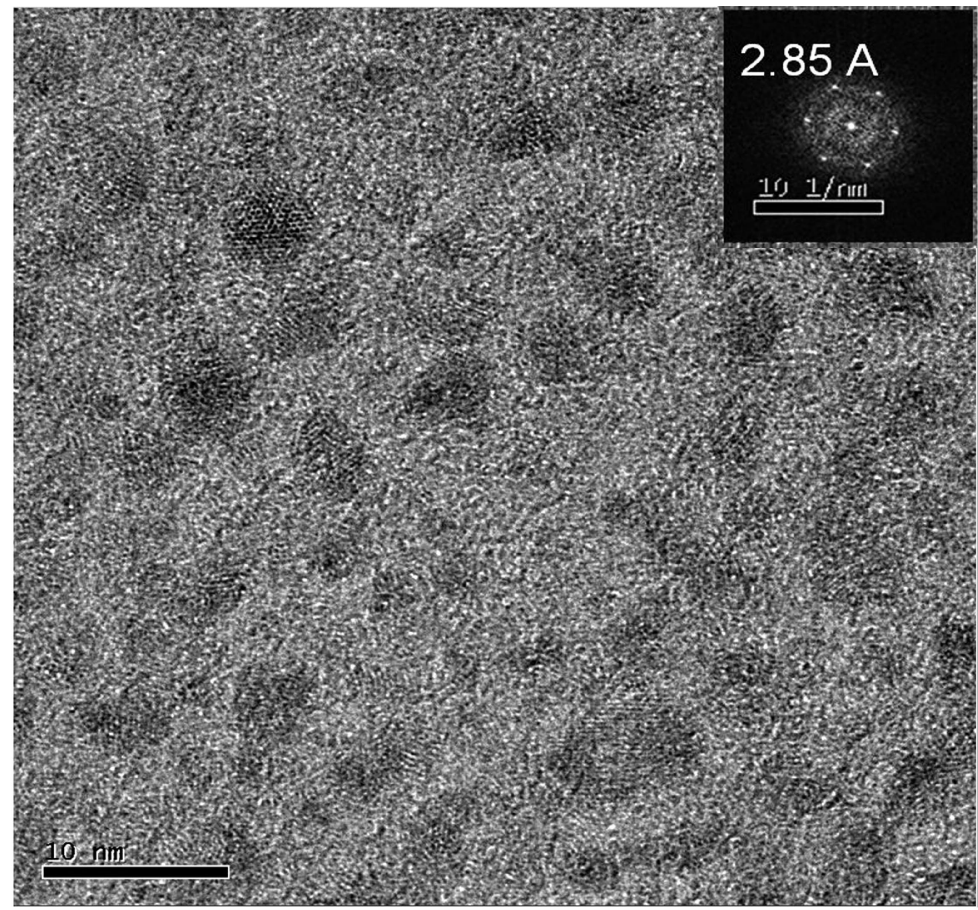

(b)

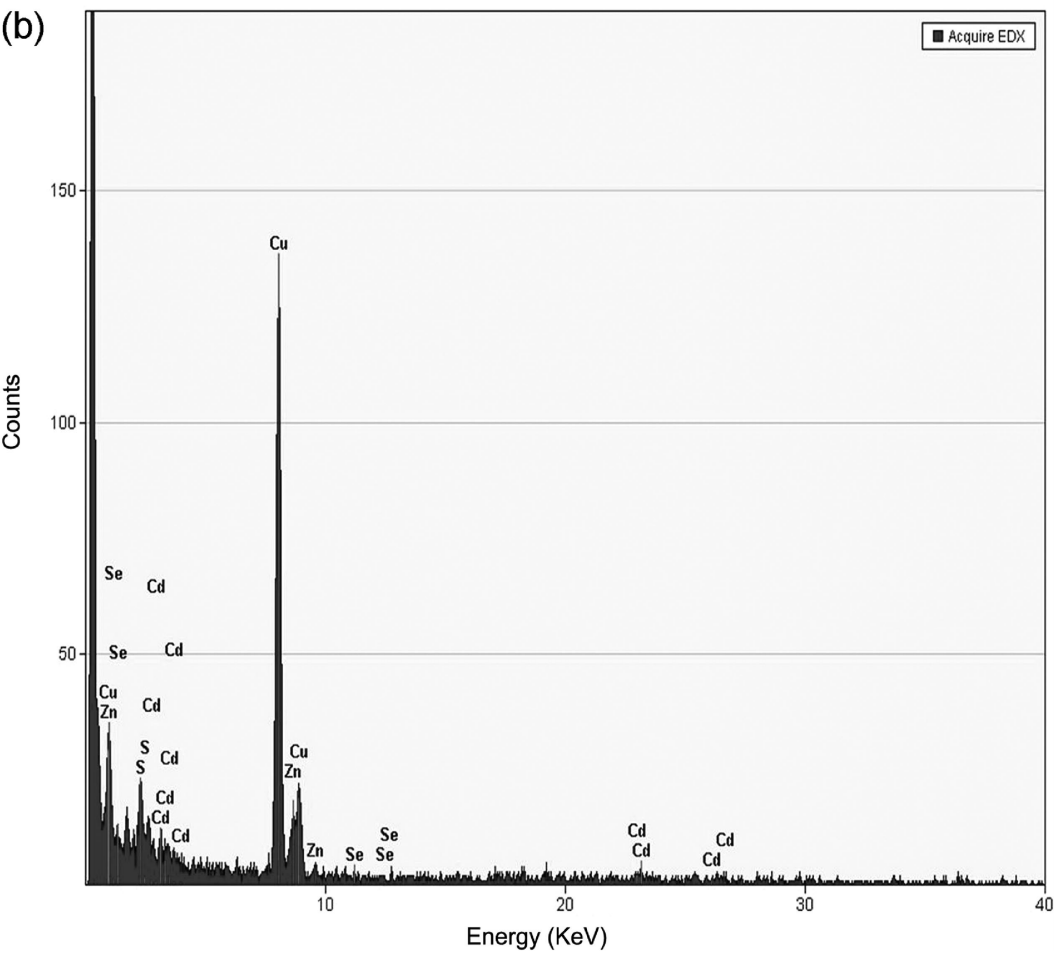

Figure 1. (a) HRTEM of CdSe/ZnS core-shell QDs revealing lattice fringes. Inset shows the corresponding FFT image of a particular QD illustrating highly crystalline structure of QDs. (b) EDAX spectrum of CdSe/ZnS QDs.

contribution to the lifetime $\tau$. We have used the amplitude weighted time constants for $\tau_{\mathrm{D}}$ and $\tau_{\mathrm{DA}}$ to evaluate the energytransfer efficiency $(E)$ using eq 3.

\section{Results and Discussion}

Figure 1a shows the HRTEM micrograph of CdSe/ZnS core-shell QDs, which reveals particles with a honeycomb structures having an average size of $4-4.5 \mathrm{~nm}$. The existence of lattice fringes on the HRTEM image illustrates the highly crystalline nature of core-shell QDs. The fast Fourier transform (FFT) pattern from a single QD (inset of Figure 1a) reveals several well-defined sharp spots forming a hexagonal structure. The spots are characterized by an interplanar distance $(d)$ of $2.85 \AA^{\prime}$, which might be assigned to the 101 plane of hexagonal wurtzite structure for the QDs. ${ }^{21} \mathrm{~A}$ typical EDAX spectrum (Figure 1b) from the QDs confirms that they are composed of $\mathrm{Cd}$ and Se with an atomic ratio of $\sim 1: 1$ together with $\mathrm{Zn}$ and $\mathrm{Se}$. The $\mathrm{Cu}$ signal is attributed to the carbon-coated copper grid. It has to be mentioned that the vendor, Evident Technologies, measures the size of QDs using a Malvern zetasizer, which includes the size of the core, shell, and ligand. We tried to measure the shell thickness in the TEM image of core-shell 

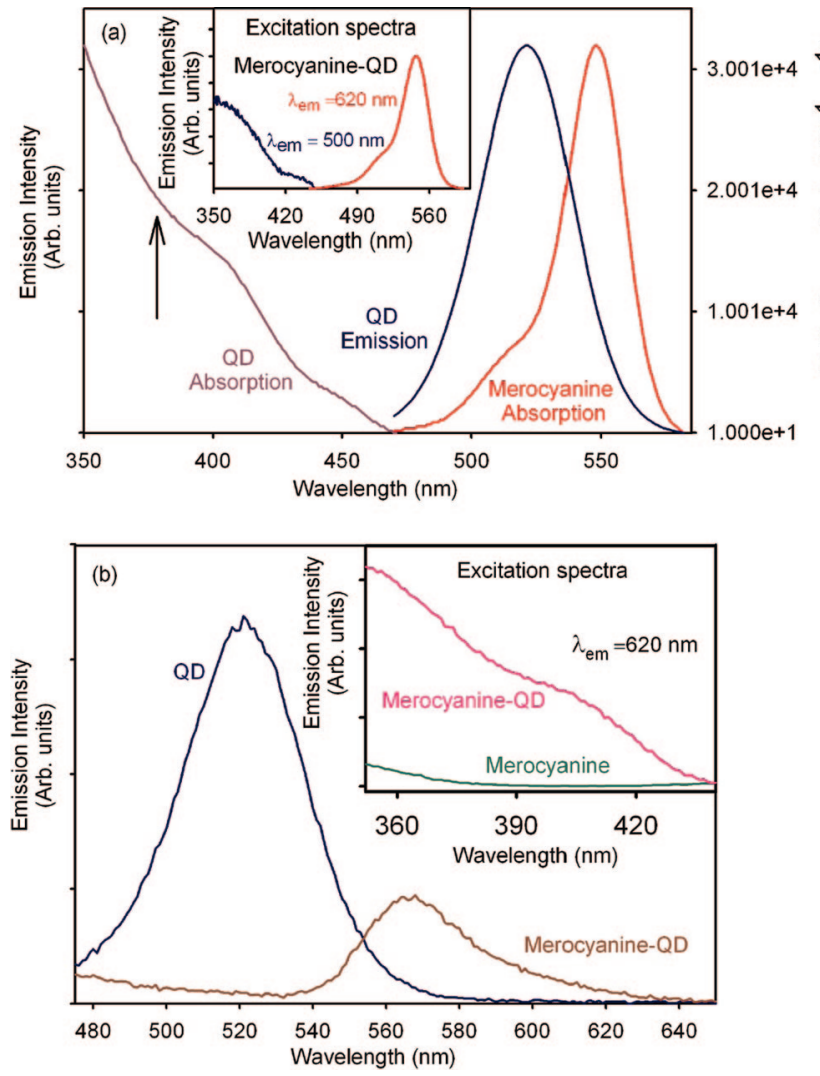

Figure 2. (a) Steady-state absorption spectrum of CdSe/ZnS core-shell QDs together with the spectral overlap between emission spectrum of QDs and the absorption spectrum of MC540. The inset shows the excitation spectra of QD-MC540 conjugate at $\lambda_{\mathrm{em}}=500$ and $620 \mathrm{~nm}$. The arrow indicates the excitation wavelength $(375 \mathrm{~nm})$ used to excite QDs. (b)The 375-nm laser light is used for the exclusive excitation of QDs in the conjugates, and the observed peak at $570 \mathrm{~nm}$ corresponding to the MC540 emission occurs as a result of FRET from the QDs. Inset shows the excitation spectra of MC540 and QD-MC540 conjugate at $\lambda_{\mathrm{em}}=620 \mathrm{~nm}$.

QDs but failed probably due to the very small thickness of the shell $(\sim 0.3 \mathrm{~nm})$.

Figure $2 \mathrm{a}$ illustrates the absorption spectrum of QDs in toluene together with the spectral overlap between the emission spectrum of QDs and the absorption spectrum of the MC540 in toluene. The huge spectral overlap suggests that an efficient FRET between the donor (QDs) and the acceptor (MC540) can take place when MC540 binds to the surface of QDs. For the energy-transfer studies, the broad absorption spectrum of the QDs (Figure 2a) enabled us to find a suitable wavelength where the donor QDs could be selectively excited without the acceptor drug getting appreciably excited. The arrow in the absorption spectrum of QDs in Figure 2a indicates the excitation wavelength used to excite QDs (i.e., $375 \mathrm{~nm}$ ). It has to be noted that the excitation spectra of QD-MC540 complex at the emission peak of QDs $(500 \mathrm{~nm})$ and MC540 $(620 \mathrm{~nm})$ matches with their corresponding absorption spectra (inset of Figure 2a) indicating negligible perturbation in the spectroscopic properties of either QDs or MC540 in the QD-MC540 complex.

It has been reported that FRET can be an efficient process between QDs and dyes, including photosensitizers. ${ }^{4}$ Figure $2 \mathrm{~b}$ reveals a significant quenching in the steady-state PL intensity of the QDs (emission peak $520 \mathrm{~nm}$ ) in the QD-drug complex on exciting at $375 \mathrm{~nm}$. This was accompanied by an increase in the fluorescence of MC540 (emission peak $570 \mathrm{~nm}$ ) as a result of FRET. These observations are confirmed by fluorescence
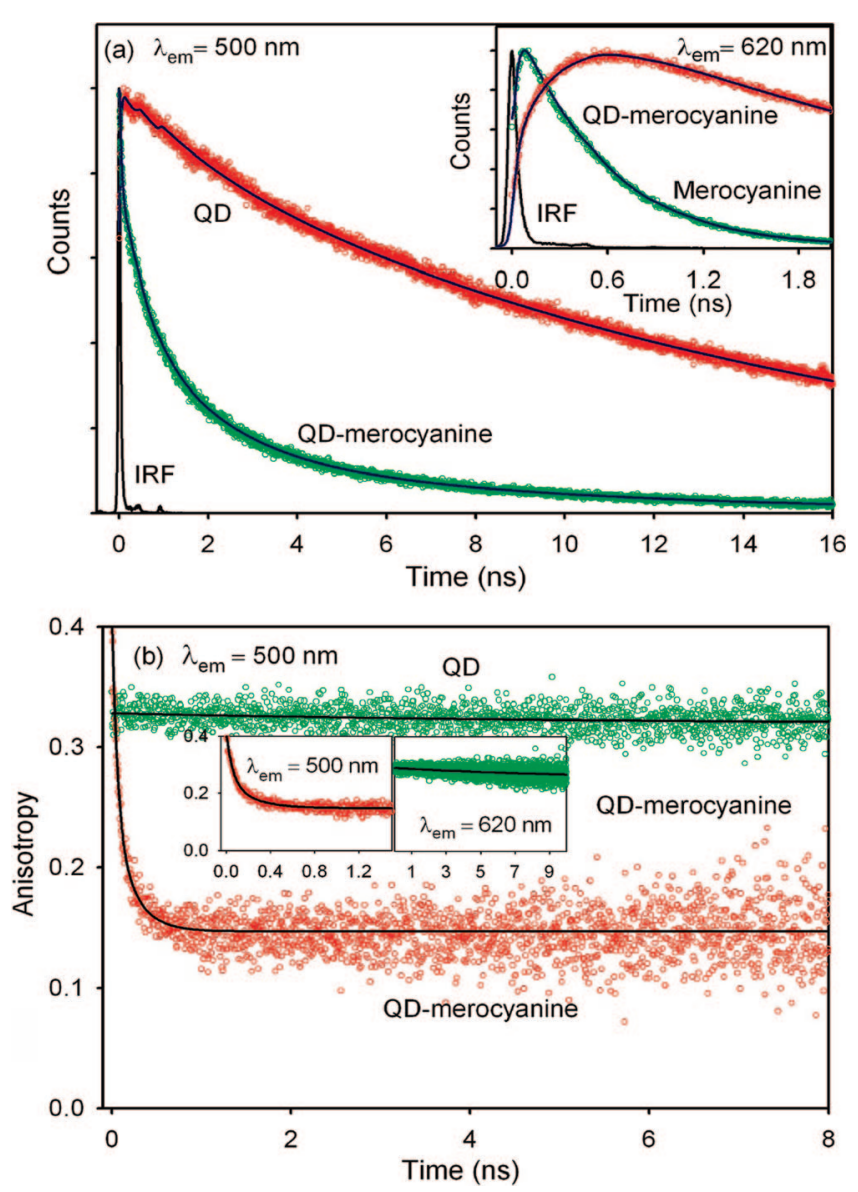

Figure 3. (a) Picosecond-resolved PL transients (IRF $=80 \mathrm{ps}$ ) of QD in the absence and presence of MC540 at $\lambda_{\mathrm{em}}=500 \mathrm{~nm}$. The inset shows the picosecond-resolved PL transients of MC540 and QD-MC540 conjugate at $\lambda_{\mathrm{em}}=620 \mathrm{~nm}$, clearly revealing a rise component for the QD-MC540, which is completely absent in the transient of MC540 alone. (b) Picosecond-resolved PL anisotropy of QD and QD-MC540 at $\lambda_{\mathrm{em}}=500 \mathrm{~nm}$. The inset shows the picosecond-resolved PL anisotropy of QD-MC540 at $\lambda_{\mathrm{em}}=500 \mathrm{~nm}$ (left) in a time window of $1.5 \mathrm{~ns}$ and at $\lambda_{\mathrm{em}}=620 \mathrm{~nm}$ (right) in a time window of $10 \mathrm{~ns}$.

lifetime measurements, where a substantial shortening of the QD donor lifetime is measured in the presence of the drug (Figure 3a). However, the quenching observed here could be due to either energy transfer or other nonradiative processes ${ }^{14}$ between QDs and the drug molecules. This is easily verified by monitoring the lifetime components of QD-MC540 at the emission peak of acceptor. As shown in the inset of Figure 3a, QD-MC540 at $620 \mathrm{~nm}$ shows a clear signature of a rise

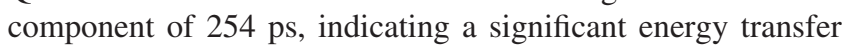
from QDs to the drug via FRET. It is expected that the QD emission at $520 \mathrm{~nm}$ is transferred nonradiatively to MC540 through space dipole-dipole coupling leading to detection of sensitized emission from the drug at $570 \mathrm{~nm}$. The signature of the sensitized emission from the drug is also borne out from the excitation spectrum of QD-MC540 monitored at $620 \mathrm{~nm}$ (inset of Figure 2b). Upon monitoring emission of QD-MC540 at $620 \mathrm{~nm}$, which solely originates from the MC540 emission, the excitation spectrum is found to be identical with the absorption profile of the free QDs (inset of Figure 2b). No such signature is found in the excitation spectrum of MC540 alone clearly indicating the sensitized emission from acceptor.

The significantly large spectral overlap of the QDs (donor) with that of the conjugated drug molecule (acceptor) allowed us to carry out FRET studies to estimate the donor-acceptor 
distance in QD-MC540 through picosecond-resolved measurements performed using a 375-nm excitation laser. The picosecondresolved transients of QDs (donor) alone at $\lambda_{\mathrm{em}}=500 \mathrm{~nm}$ reveals multiexponential decay ${ }^{22}$ with an average time constant of $12 \mathrm{~ns}$ compared to $0.6 \mathrm{~ns}$ for the conjugate system. This gives a FRET efficiency of $95 \%$ and a donor-acceptor distance of $25 \AA$. This is consistent with the report by Weiss et al. ${ }^{11}$ concerning the FRET studies between peptide-coated greenemitting QDs and rose bengal, where they reported a drastic shortening of the fluorescence lifetime of QDs upon conjugation to rose bengal. Moreover, the FRET efficiency obtained by them $(>90 \%)$ closely matches with our results $(95 \%)$. It is known that the diameter of the CdS core is $21 \AA$ and the thickness of shell is $\sim 3 \AA$ (provided by Evident Technologies). Also, the size of the TOPO molecule around the QD is $12 \AA$. Taken together, the radius of the TOPO-capped core-shell QD will be $\sim 26 \AA$. The estimated donor-acceptor distance of $\sim 26 \AA$ in the QD-MC540 complex clearly supports our model of direct binding of drug molecule to the surface of $\mathrm{CdSe} / \mathrm{ZnS}$ QDs, making the FRET process efficient (Scheme 2).

We have further probed the QD-MC540 system by undertaking the time-resolved anisotropy measurements. Figure $3 \mathrm{~b}$ shows the picosecond-resolved PL anisotropy decays of QDs and QD-MC540 at $500 \mathrm{~nm}$. QDs in toluene (500 nm) reveal anisotropy decay with a rotational time constant of 8 ns. On the other hand, QD-MC540 revealed a major contribution of fast component of $130 \mathrm{ps}$ together with a minor contribution of a slow component of $8 \mathrm{~ns}$. The observation of a higher contribution of fast component $(130 \mathrm{ps})$ in the conjugate indicates the exciton migration in the QDs, which transfers its energy to the proximal drug molecules through dipole-dipole coupling leading to a strong sensitized emission from the drug. Using the time-resolved fluorescence anisotropy study, Ghiggino and co-workers ${ }^{17}$ have demonstrated the dynamics of energy transfer in multiporphyrin functionalized dendrimers where they observed a fast decay in the anisotropy profile before leveling off to a nonzero residual anisotropy similar to our observation. They explained their observation to the ability of these molecules to absorb light and then efficiently delocalize the excitation energy over the chromophore arrays during the energy migration process. The left inset of Figure $3 \mathrm{~b}$ shows picosecond-resolved anisotropy decay of QD-MC540 at $500 \mathrm{~nm}$ in a small time window. The anisotropy decay of QD-MC540 at $620 \mathrm{~nm}$ (right inset of Figure $3 b$ ) reveals a very slow component that does not decay within our experimental window, characteristic of drug bound to QDs. Using the hydrodynamic diameter, we also calculated the rotational correlation time $\left(\tau_{\mathrm{r}}\right)$ of QDs using the Stokes-Einstein-Debye theory and found a value of $9.5 \mathrm{~ns}$, close to the experimentally obtained value and consistent with our report on a similar system. ${ }^{23}$ The observation of a fast decay component in the anisotropy of QD-MC540 complex in contrast with the slow decay component in the anisotropy of QD alone provides strong evidence that FRET occurs between QDs and drug molecules.

\section{Conclusions}

An efficient FRET between CdSe/ZnS QDs (donor) and an anionic drug Merocyanine 540 (acceptor) has been demonstrated through steady-state and time-resolved studies. The FRET resulted in a strong sensitized emission of the drug, which is clearly manifested in the QD-drug complex through a fast component in the PL decay of the donor followed by a rise component of similar time scale for the acceptor. The estimated donor-acceptor distance from FRET measurements clearly indicates that the drug molecules are directly bound to the surface of QDs, possibly through the attachment of the sulfur of MC540 to the $\mathrm{ZnS}$ shell by replacing TOPO ligands from the outer surface of QDs. The observation of a fast decay component in the fluorescence anisotropy of QD-MC540, which is attributed to the migration of dipolar exciton from QDs to the proximal drug molecules, also supports the FRET. It is anticipated that the described methodology of sensitized emission from a QD-drug complex could be applied to other systems as well for the confirmation of absolute sensitization of other drugs.

Acknowledgment. S.S.N. thanks CSIR, India for a fellowship. We thank DST for financial grant (SR/FTP/PS-05/2004).

\section{References and Notes}

(1) Alivisatos, A. P. Science 1996, 271, 933.

(2) Klarreich, E. Nature 2001, 413, 450.

(3) Alivisatos, P. Nat. Biotechnol. 2004, 22, 47.

(4) Samia, A. C. S.; Chen, X.; Burda, C. J. Am. Chem. Soc. 2003, $125,15736$.

(5) Samia, A. C. S.; Dayal, S.; Burda, C. Photochem. Photobiol. 2006, $82,617$. 6278 .

(6) Shi, L.; Hernandez, B.; Selke, M. J. Am. Chem. Soc. 2006, 128,

(7) Bakalova, R.; Ohba, H.; Zhelev, Z.; Ishikawa, M.; Baba, Y. Nat. Biotechnol. 2004, 22, 1360.

(8) Dayal, S.; Burda, C. J. Am. Chem. Soc. 2008, 130, 2890.

(9) Dayal, S.; Burda, C. J. Am. Chem. Soc. 2007, 129, 7977.

(10) Dayal, S.; Lou, Y.; Samia, A. C. S.; Berlin, J. C.; Kenney, M. E.; Burda, C. J. Am. Chem. Soc. 2006, 128, 13974.

(11) Tsay, J. M.; Trzoss, M.; Shi, L.; Kong, X.; Selke, M.; Jung, M. E.; Weiss, S. J. Am. Chem. Soc. 2007, 129, 6865-6871.

(12) Hsieh, J.-M.; Ho, M.-L.; Wu, P.-W.; Chou, P.-T.; Tsai, T.-T.; Chi, Y. Chem. Commun. 2006, 615.

(13) Hering, V. R.; Gibson, G.; Schumacher, R. I.; Faljoni-Alario, A.; Politi, M. J. Bioconjugate Chem. 2007, 18, 1705.

(14) Burda, C.; Chen, X.; Narayanan, R.; El-Sayed, M. A. Chem. Rev. $\mathbf{2 0 0 5}, 105,1025$.

(15) Hamer, F. M. The Chemistry of Heterocyclic Compound. The Cyanine Dyes and Related Compounds; Interscience: New York, 1963.

(16) Varnavski, O. P.; Ranasinghe, M.; Yan, X.; Bauer, C. A.; Chung, S.-J.; Perry, J. W.; Marder, S. R.; Goodson, T., III J. Am. Chem. Soc. 2006, $128,10988$.

(17) Yeow, E. K. L.; Ghiggino, K. P.; Reek, J. N. H.; Crossley, M. J.; Bosman, A. W.; Schenning, A. P. H. J.; Meijer, E. W. J. Phys. Chem. B 2000, 104, 2596.

(18) Khazraji, A. C.; Hotchandani, S.; Das, S.; Kamat, P. V. J. Phys. Chem. B 1999, 103, 4693.

(19) O'Connor, D. V.; Philips, D. Time correlated single photon counting; Academic Press: London, 1984.

(20) Lakowicz, J. R. Principles of fluorescence spectroscopy; Kluwer Academic/Plenum: New York, 1999.

(21) Cheng, Y.; Wang, Y.; Bao, F.; Chen, D. J. Phys. Chem. B 2006, 110,9448 .

(22) Bawendi, M. G.; Carroll, P. J.; Wilson, W. L.; Brus, L. E. J. Chem. Phys. 1992, 96, 946.

(23) Narayanan, S. S.; Sarkar, R.; Sinha, S. S.; Dias, F.; Monkman, A.; Pal, S. K. J. Phys. Chem. C 2008, 112, 3423.

JP8032534 